\title{
Time to Make the Most Use of Three-Dimensional Global Strains in Daily Clinical Practice
}

\author{
Akira Yamada, MD, PhD
}

$\mathbf{L}$ eft ventricular ejection fraction (LVEF) and global longitudinal strain (GLS) are cardiac parameters reflecting LV systolic function. It is possible to identify reduced LVEF with GLS, ${ }^{1}$ but the correlation of LVEF and GLS is rather weak, and global circumferential strain (GCS) has a stronger correlation with LVEF than GLS. ${ }^{2}$ Chan et al reported that subendocardial myocardial infarction (MI) is associated with a significant reduction in longitudinal strain despite relatively preserved circumferential strain, whereas transmural infarction is associated with a reduction of both LS and CS. ${ }^{3}$ Their research clearly explained the difference in clinical implications between GLS and GCS; that is, GLS is more sensitive to subendocardial ischemia. GLS is now accepted as a marker of subclinical LV dysfunction beyond LVEF in various clinical situations, such as estimating cardiac toxicity in cancer chemotherapy. ${ }^{4}$ Furthermore, GLS is reported to be a better predictor of prognosis than LVEF in heart failure with reduced LVEF (HFrEF). ${ }^{5}$ Iwahashi et al reported that three dimensional (3D)-GLS measured immediately after ST-elevation MI (STEMI) is independently associated with LV remodeling as well as 1-year prognosis. ${ }^{6}$ In addition, in this issue of the Journal, they examine the prognostic utility of $3 \mathrm{D}$ global strains for the prediction of 10 -year prognosis in $1^{\text {st-STEMI patients. }}{ }^{7}$ They show that 3 D-GLS was the strongest predictor for major adverse cardiac events, and its combination with 3D-GCS could predict them more accurately. It is a surprising and beneficial finding that strain measurements in the acute phase can predict such a long-term outcome.

Two-dimensional (2D)- and 3D-GLS have a strong correlation as reported: ${ }^{1}$ however, there are several limitations in global strain measurements with 2D speckle tracking echocardiography (STE). In 2D STE, each image for the strain measurement is recorded separately in different cardiac cycles, which may influence the global strain values, especially under unstable cardiac condition. Moreover, "through-plane motion" in 2D STE can be a problem (Figure). It produces discrepancies in the CS measurements, particularly at the LV basal level. ${ }^{8}$ On the other hand, 3D full-volume acquisition of LV data will, in theory, overcome these limitations. Additionally, time from image acquisition to postprocessing analysis is significantly reduced with

\section{Article p1735}

3D STE compared with $2 \mathrm{D}$ STE, ${ }^{1}$ which is one of the practical merits of 3D STE.

One of the key points to bear in mind is that GLS is merely an indicator of LV performance. There are clinical parameters, other than LV function, that are also associated with prognosis in STEMI patients. For example, mitral regurgitation (MR) is a common complication after STEMI, and it is known that MR is related to a poorer prognosis. ${ }^{9}$ MR itself plays a role in reducing LV afterload, which may contribute to seemingly increased GLS. On top of that, for an inferior MI with right ventricular (RV) infarction, decreased RV systolic function is a major risk factor for death, sudden death, HF, and stroke after MI, ${ }^{10}$ but obviously the LV GLS does not include RV function. It is important to take various parameters into account in addition to GLS in a comprehensive way to predict the outcomes in STEMI patients.

According to the guidelines for acute coronary syndrome (ACS) announced by the Japanese Circulation Society, echocardiography is recommended as Class I to estimate $\mathrm{LV} / \mathrm{RV}$ function and to detect LV wall motion abnormality, mural thrombus and mechanical complications in ACS patients. ${ }^{11}$ Yet reperfusion therapy needs to be performed as soon as possible once the diagnosis of STEMI is confirmed, and echocardiography examination cannot be justified if it leads to a delay in reperfusion therapy. Even so, echocardiography including 3D STE should be performed at least within $48 \mathrm{~h}$ of admission in STEMI patients, as shown in the studies of Iwahashi et al.6,7 Performing echocardiography is possible even at the bedside, and it is clinically valuable not only to examine cardiac function and possible complications but also to predict the patient's short-term as well as long-term prognosis for a better therapeutic strategy.

MI is a major cardiac disease causing HFrEF. If poorer prognosis is indicated by $3 \mathrm{D}$ strain measurements, it is important to make use of them for more suitable patient care to improve the prognosis. Recently, some novel agents for the treatment of HFrEF have become clinically available such as sodium-glucose cotransporter-2 inhibitor,

The opinions expressed in this article are not necessarily those of the editors or of the Japanese Circulation Society.

Received June 7, 2021; accepted June 7, 2021; J-STAGE Advance Publication released online July 9, 2021

Department of Cardiology, Fujita Health University School of Medicine, Toyoake, Japan

Mailing address: Akira Yamada, MD, PhD, Department of Cardiology, Fujita Health University School of Medicine, 1-98

Dengakugakubo, Kutsukake-cho, Toyoake 470-1192, Japan. E-mail: a-yamada@fujita-hu.ac.jp

All rights are reserved to the Japanese Circulation Society. For permissions, please e-mail: cj@j-circ.or.jp

ISSN-1346-9843 


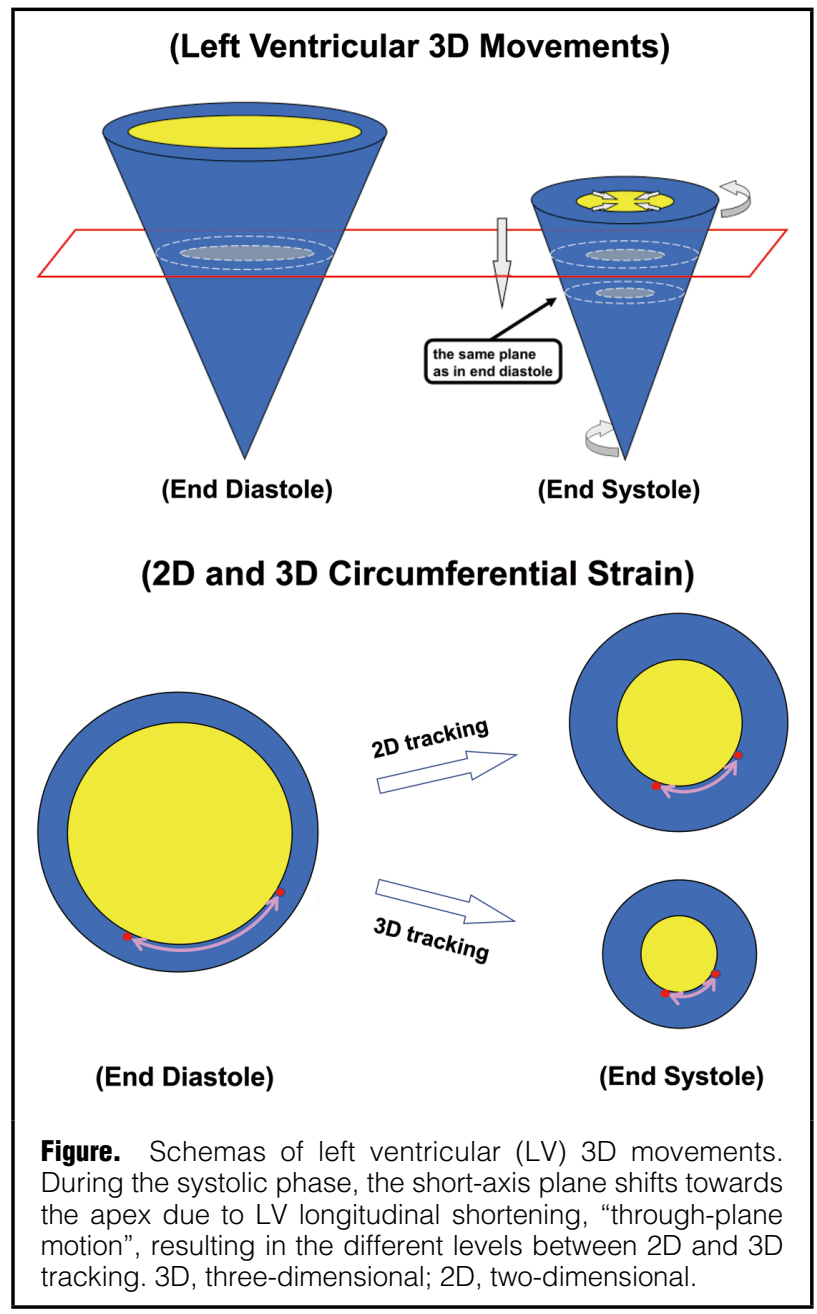

$\mathrm{HCN}$ channel blocker, and angiotensin-receptor neprilysin inhibitor. Further research is expected to clarify whether more aggressive therapeutic intervention, including these drugs, alters follow-up 3D strain values and improve prognosis in STEMI patients with poor prognosis suggested by initial 3D strain measurements.

It is true that in 3D STE there are still some issues to be resolved, such as inter-vendor discrepancies of strain values; nevertheless, it is time to measure 3D strains and make the most use of them in daily clinical practice, particularly to collect prognostic information. The research of Iwahashi et $\mathrm{al}^{7}$ presented in this issue of the Journal is a milestone towards the new era of 3D STE for clinical practice.

\section{References}

1. Luis SA, Yamada A, Khandheria BK, Speranza V, Benjamin A, Ischenko M, et al. Use of three-dimensional speckle-tracking echocardiography for quantitative assessment of global left ventricular function: A comparative study to three-dimensional echocardiography. J Am Soc Echocardiogr 2014; 27: 285-291.

2. StokkeTM, Hasselberg NE, Smedsrud MK, Sarvari SI, Haugaa $\mathrm{KH}$, Smiseth OA, et al. Geometry as a confounder when assessing ventricular systolic function: Comparison between ejection fraction and strain. J Am Coll Cardiol 2017; 70: 942-954.

3. Chan J, Hanekom L, Wong C, Leano R, Cho GY, Marwick TH. Differentiation of subendocardial and transmural infarction using two-dimensional strain rate imaging to assess short-axis and long-axis myocardial function. J Am Coll Cardiol 2006; 48: 2026-2033.

4. Zamorano JL, Lancellotti P, Muñoz DR, Aboyans V, Asteggiano R, Galderisi M, et al. 2016 ESC Position Paper on cancer treatments and cardiovascular toxicity developed under the auspices of the ESC Committee for Practice Guidelines: The Task Force for cancer treatments and cardiovascular toxicity of the European Society of Cardiology (ESC). Eur Heart J 2016; 37: 2768-2801.

5. Sengeløv M, Jørgensen PG, Jensen JS, Bruun NE, Olsen FJ, Fritz-Hansen T, et al. Global longitudinal strain is a superior predictor of all-cause mortality in heart failure with reduced ejection fraction. JACC Cardiovasc Imaging 2015; 8: $1351-$ 1359.

6. Iwahashi N, Kirigaya J, Abe T, Horii M, Toya N, Hanajima Y, et al. Impact of three-dimensional global longitudinal strain for patients with acute myocardial infarction. Eur Heart J Cardiovasc Imaging, doi:10.1093/ehjci/jeaa241.

7. Iwahashi N, Kirigaya J, Gohbara M, Abe T, Horii M, Hanajima $\mathrm{Y}$, et al. Global strain measured by three-dimensional speckle tracking echocardiography is a useful predictor for 10-year prognosis after a first ST-elevation acute myocardial infarction. Circ $J$ 2021; 85: $1735-1743$.

8. Wu VC, Takeuchi M, Otani K, Haruki N, Yoshitani H, Tamura $\mathrm{M}$, et al. Effect of through-plane and twisting motion on left ventricular strain calculation: Direct comparison between twodimensional and three-dimensional speckle-tracking echocardiography. J Am Soc Echocardiogr 2013; 26: 1274-1281.

9. Grigioni F, Enriquez-Sarano M, Zehr KJ, Bailey KR, Tajik AJ. Ischemic mitral regurgitation: Long-term outcome and prognostic implications with quantitative Doppler assessment. Circulation 2001; 103: 1759-1764.

10. Anavekar NS, Skali H, Bourgoun M, Ghali JK, Kober L, Maggioni AP, et al. Usefulness of right ventricular fractional area change to predict death, heart failure, and stroke following myocardial infarction (from the VALIANT ECHO study). Am J Cardiol 2008; 101: 607-612.

11. Kimura K, Kimura T, Ishihara M, Nakagawa Y, Nakao K, Miyauchi K, et al. JCS 2018 guideline on diagnosis and treatment of acute coronary syndrome. Circ J 2019; 83: 1085-1196. 